Journal of Vibration and Control 2020, Vol. 26(15-16) II55-1164

\title{
A novel design of positive position feedback controller based on maximum damping and $\mathrm{H}_{2}$ optimization
}

\author{
Ahmad Paknejad'®, Gouying Zhao', Michel Osée' $\odot$, \\ Arnaud Deraemaeker ${ }^{2}$, Frédéric Robert' ${ }^{1}$ and \\ Christophe Collette ${ }^{1,3}$
}

\begin{abstract}
Positive position feedback is an attractive control law for the control of plants having no high frequency roll-off. The tuning of the parameters of the positive position feedback to obtain the desired closed-loop performance is quite challenging. This paper presents a technique to design the positive position feedback controller with the optimal damping. The technique is demonstrated on a single degree-of-freedom system. The poles of the positive position feedback are tuned using the method of maximum damping, which states that the maximum damping is achieved when both closed-loop poles of the system are merged. The parameters of the positive position feedback are dependent on the desired target damping in the closed-loop system. However, arbitrary choice of target damping results in high response at the frequencies lower than the tuning frequency. The optimal value of the target damping is obtained by minimizing the $\mathrm{H}_{2}$ norm of the closed-loop transfer function of the system. The influence of the various parameters of the positive position feedback on the closedloop response of the system is also studied. Finally, the experiments are conducted to verify the effectiveness of the proposed technique.
\end{abstract}

\section{Keywords}

Active vibration control, smart structure, maximum damping criterion, $\mathrm{H}_{2}$ optimization, positive position feedback

\section{Introduction}

Lightweight structures are usually preferred in engineering applications for reducing cost and power consumption (Naji et al., 2014). These structures have a low inherent structural damping (Fuller and Von Flotow, 1995), which potentially leads to fatigue problem (Lazan, 1954; Pota et al., 1999) and noise radiation (Oude Nijhuis, 2003; Wilby and Pope, 1980). This has concern for aerospace structures where the fail-safe design methodology is usually adopted (Eaton, 1997; Inman, 2001; Osinski, 2018). Therefore, an effective technique is required for damping structural vibrations. Direct velocity feedback (Balas, 1979), integral force feedback (Preumont et al., 1992), digital shunt absorber (Fleming et al., 2000) and positive position feedback (PPF) (Fanson, 1987) are some of the methods that have been used extensively for increasing the damping of structural resonance. Among them, the PPF, as a second order low-pass filter, is one of the effective control techniques to be implemented on the plant having no high frequency roll-off. It was introduced by Goh (1983).

Some earlier studies (Dosch et al., 1992a, 1992b; Fagan, 1993) suggested to use a factor of 1.3 or 1.45 as the ratio between the frequency corresponding to the pole of the controller and the resonance frequency of the primary system. The suggested value for the damping ratio of the pole was between 0.01 and 0.5 . Designing the PPF to achieve a desired damping in the closed-loop response was a major concern. Kwak and Han (1998) studied the potential of using a genetic algorithm to find the optimal parameters of the PPF. A serious weakness with this argument, however, is that the feedback gain and the damping ratio of the pole of the PPF were kept constant. Fenik and Starek (2008) and McEver (1999) studied the optimal PPF based on the

\footnotetext{
'BEAMS Department, Université Libre de Bruxelles, Belgium ${ }^{2}$ BATir Department, Université Libre de Bruxelles, Belgium

${ }^{3}$ Department of Aerospace and Mechanical Engineering, Université de Liége, Belgium

Received: 9 May 2019; accepted: I October 2019
}

\section{Corresponding author:}

Ahmad Paknejad, Precision Mechatronics Laboratory (PML), BEAMS Department, Université Libre de Bruxelles, CP 194/02, 50 F. D. Roosevelt Ave., 1050 Brussels, Belgium.

Email: ahmad.paknejad@ulb.ac.be 
method of maximum damping. The main shortcoming of their studies was that the parameters were optimized for a given value of the feedback gain. Dosch et al. (1992a, 1992b) defined a cost function in the time domain to optimize the frequency and the damping ratio of the PPF. The optimization tries to achieve the trade-off between minimizing the impulse response and minimizing the control effort. These optimization approaches have been focused on the damping of the resonance.

Another major concern in the design of the PPF parameters is the increased magnitude of the closed-loop response at the frequencies lower than the tuning frequency. Although this phenomenon will not destabilize the system if the feedback gain is chosen appropriately, reducing its effect will greatly enhance the performance of the system. One way to reduce such effect is to transform the classical PPF compensator into fractional format (Marinangeli et al., 2018; Niu et al., 2018).

There has been scant research in the area of optimal PPF. This study attempts to fill in this gap. A technique is proposed to design the PPF controller based on a combination of optimal damping and $H_{2}$ optimization. The controller maximizes the damping and minimizes the magnitude of response of the closed-loop system. First, the method of maximum damping criterion is used to find the ideal location of the pole of the controller. The ideal location of the pole is not unique and is dependent on the damping of the closed-loop system. The optimal value of the damping is obtained through optimization. In the optimization problem, the damping of the closed-loop system is taken as the design variable, while the objective is the minimization of the $\mathrm{H}_{2}$ norm of the closed-loop transfer function.

The rest of the paper is organized as follows. Section 2 describes the system considered and the technique for designing the optimal PPF controller. After that, the sensitivity analysis of the PPF parameters is conducted in Section 3. This is followed by the experimental validation of the technique in Section 4. Finally, the results and conclusions are presented in Section 5.

\section{Mathematical modeling and $\boldsymbol{H}_{\mathbf{2}}$ optimization}

An undamped single degree-of-freedom (DOF) system with a mass $m$ and a stiffness $k$ is considered as the primary structure (Figure 1). It is excited by an external force $f_{d}$. The active control system consists of an absolute displacement sensor $x$, mounted on the mass, the PPF controller and an actuator $f_{a}$. The governing equations of motion are written as

$$
\begin{gathered}
m \ddot{x}+k x=f_{d}+f_{a} \\
f_{a}=g_{f} u
\end{gathered}
$$

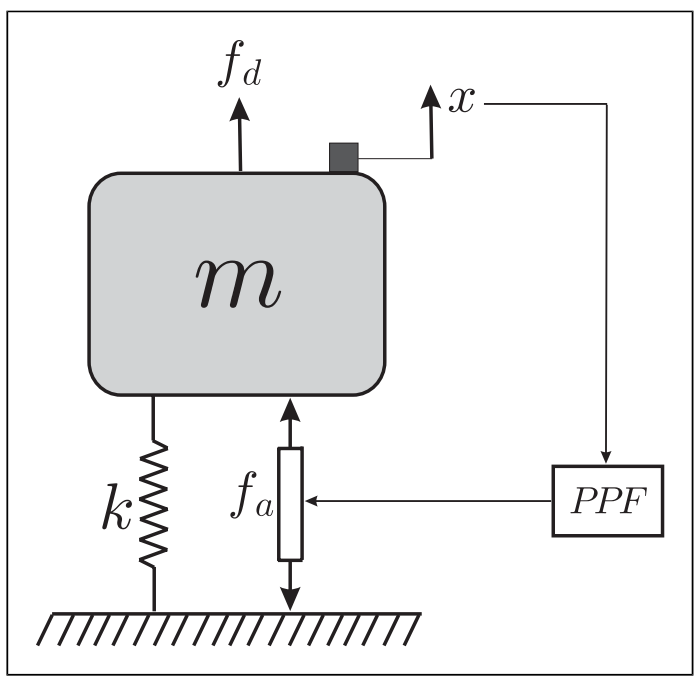

Figure I. Schematic of the system under consideration consisting of a single degree-of-freedom oscillator combined with the active control part.

and the dynamics of the controller read

$$
\ddot{u}+2 \xi_{f} \omega_{f} \dot{u}+\omega_{f}^{2} u=\omega_{f}^{2} x
$$

where $u, \xi_{f}, \omega_{f}$ and $g_{f}$ are the control signal, the damping ratio, the frequency and the gain of the controller, respectively. The above equations are normalized with respect to the dimensionless time $\tau=\omega_{0} t$, where $\omega_{0}=\sqrt{k / m}$, as

$$
\begin{gathered}
x_{1}^{\prime \prime}+x_{1}=f+\beta x_{2} \\
x_{2}^{\prime \prime}+2 \xi \alpha x_{2}^{\prime}+\alpha^{2} x_{2}=\alpha^{2} x_{1}
\end{gathered}
$$

where the normalized parameters are considered as follows

$$
\begin{aligned}
& x_{1}(\tau)=x(t), \quad x_{2}(\tau)=u(t), \quad \Omega=\omega / \omega_{0}, \\
& f=\frac{1}{k} f_{d}, \quad \beta=\frac{1}{k} g_{f}, \quad \alpha=\frac{\omega_{f}}{\omega_{0}}, \quad \xi=\xi_{f}
\end{aligned}
$$

By transforming equations (3) and (4) to the Laplace domain, the transfer function of the closed-loop system from the normalized input $f$ and normalized displacement of the mass $x_{1}$ can be derived as

$$
\frac{x_{1}}{f}=\frac{s^{2}+2 \xi \alpha s+\alpha^{2}}{\left(s^{2}+1\right)\left(s^{2}+2 \xi \alpha s+\alpha^{2}\right)-\alpha^{2} \beta}
$$

where $s=j \Omega$ is the Laplace variable.

According to equation (6), the transfer function of the closed-loop system has two independent poles. The method of maximum damping is used to tune the pole of the PPF properly. It states that the best pole of the PPF is at the location where both resulting closed-loop poles have an equal damping ratio. This can be easily seen in Figure 2 for two different locations of the controller poles. The pole of 


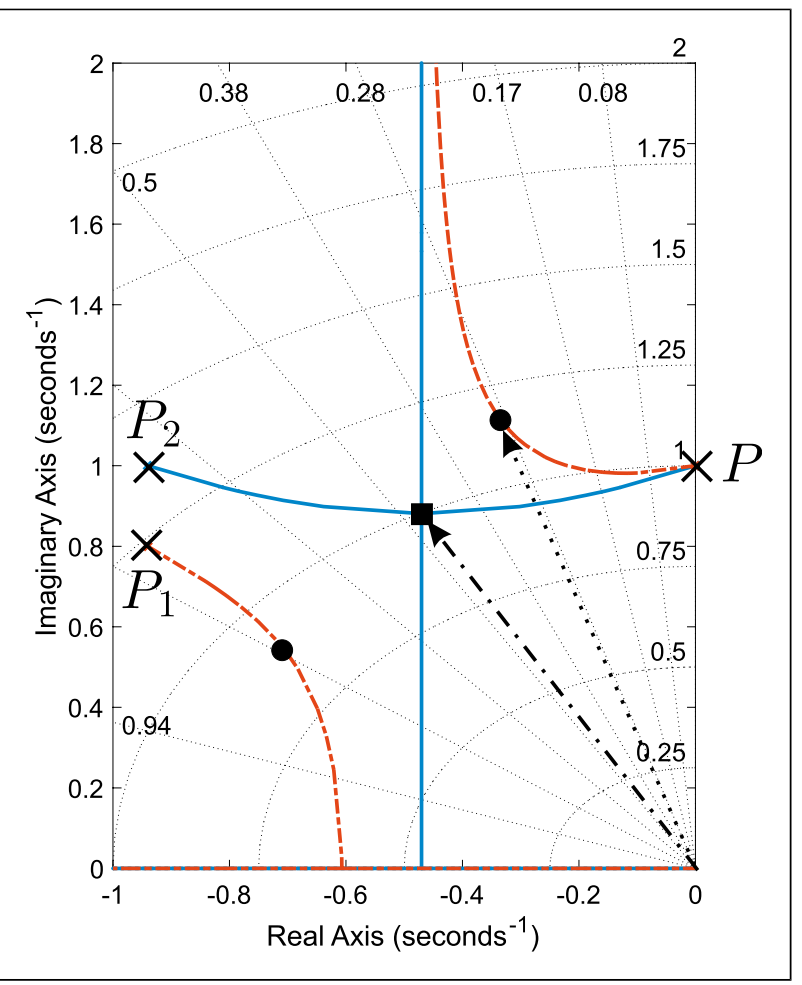

Figure 2. Root-locus curves for two different PPF controllers $\left(P_{0}\right.$ is the pole of the primary system; $\bullet$ : closed-loop poles when the pole of the PPF is placed at $P_{1}$; $\mathbf{m}$ : closed-loop poles when the pole of the PPF is placed at $P_{2}$ ). PPF: positive position feedback.

the PPF is placed at $P_{1}$ at the first attempt. The value of the closed-loop damping is about $30 \%$ as shown by the dotted arrow line. The right and the left loops are intersecting at one point when the pole of the PPF is modified to $P_{2}$. The corresponding damping ratio in this case increases to more than $45 \%$. More details on this will be given in Section 3 . By considering $\omega_{c}$ and $\eta$ as the frequency and the damping ratio corresponding to the poles of the closed-loop system, the frequency response function of the closed-loop system can be simplified as

$$
\frac{x_{1}}{f}=\frac{s^{2}+2 \xi \alpha s+\alpha^{2}}{\left(s^{2}+2 \eta \gamma s+\gamma^{2}\right)^{2}}
$$

where $\gamma=\omega_{c} / \omega_{0}$. The following equations are obtained by equating the polynomial coefficients of the denominator of the fraction on the right hand side of equations (6) and (7).

$$
\begin{gathered}
4 \eta \gamma=2 \alpha \xi \\
\left(4 \eta^{2}+2\right) \gamma^{2}=\left(\alpha^{2}+1\right) \\
4 \eta \gamma^{3}=2 \alpha \xi \\
\gamma^{4}=\alpha^{2}-\alpha^{2} \beta
\end{gathered}
$$

Table I. Parameters of the controller as a function of target closed-loop damping derived using the maximum damping criterion.

\begin{tabular}{ll}
\hline Parameters & Values \\
\hline$\alpha$ & $\sqrt{4 \eta^{2}+1}$ \\
$\beta$ & $\frac{4 \eta^{2}}{4 \eta^{2}+1}$ \\
$\xi$ & $\frac{2 \eta}{\sqrt{4 \eta^{2}+1}}$ \\
\hline
\end{tabular}

Interestingly, from equations (8a) and (8c), it can be concluded that $\gamma$ has to be equal to one to have unique and non-trivial solutions. This implies that the resonance frequency of the closed-loop system should be the same as the resonance frequency of the primary system.

$$
\omega_{c}=\omega_{0}
$$

The set of equation (8) is now simplified to three subequations. However, there are still four unknown variables, namely, $\alpha, \beta, \xi$ and $\eta$. This results in an under-determined system of equations. This is because the number of PPF controllers which can provide the merged poles in the closed-loop response is not unique. To transfer the system of equations from an under-determined to a determined system, one of the variables is set free. It is meaningful to consider the damping of the closed-loop response $\eta$ as the free variable. In such a case, the controller parameters $\alpha, \beta$ and $\xi$ are obtained for a particular value of $\eta$ in such a way that the poles of the closed-loop response are merged. As a consequence, Table 1 lists the controller parameters as a function of the target damping, $\eta$.

Therefore, the closed-loop transfer function, $x_{1} / f$, can be rewritten as

$$
\frac{x_{1}}{f}=\frac{s^{2}+4 \eta s+4 \eta^{2}+1}{\left(s^{2}+2 \eta s+1\right)^{2}}
$$

At low frequency, the magnitude of $\left(x_{1} / f\right)$ can be approximated to $\left(4 \eta^{2}+1\right)$. This means that the active damping comes at the price of the magnification of the response at low frequency, i.e. a degradation of the static stiffness. This implies that the PPF controller softens the structure for a higher value of target damping. In the view of the vibration control design, this can be a major problem. The optimal value of the damping, which minimizes the energy of the system when subjected to a Gaussian white noise input, is obtained using $\mathrm{H}_{2}$ optimization.

The square of the $\mathrm{H}_{2}$ norm of the closed-loop transfer function can be expressed as (Crandall et al., 1963)

$$
\|H\|_{2}^{2}=\int_{-\infty}^{\infty} S_{0}(\omega)|y|^{2} \omega_{0} d \Omega
$$

where $S_{0}(\omega)$ is the power spectral density of the input signal and $y$ is the transfer function between the perturbation and 
the output of the system at a given location and in a given direction. In the present study, $y$ corresponds to $x_{1} / f$. In the case of white noise excitation, $S_{0}(\omega)$ is constant as a function of frequency $\left(S_{0}(\omega)=S_{0}\right)$. Therefore, the square of $\mathrm{H}_{2}$ norm is simplified as

$$
\|H\|_{2}^{2}=S_{0} \omega_{0} \int_{-\infty}^{\infty}|y|^{2} d \Omega
$$

The minimization of the variance of the response quantity is therefore equivalent to minimizing the $\mathrm{H}_{2}$ norm of the transfer function

$$
P I=\int_{-\infty}^{\infty}|y|^{2} d \Omega
$$

According to Crandall et al. (1963), the analytical solution to the integral (equation (13)) is in the form of

$$
\begin{aligned}
& P I=\pi \frac{N_{1}+N_{2}+N_{3}+N_{4}}{D_{1}} \\
& N_{1}=\left(\frac{B_{0}^{2}}{C_{0}}\right)\left(C_{2} C_{3}-C_{1} C_{4}\right) \\
& N_{2}=C_{3}\left(B_{1}^{2}-2 B_{0} B_{2}\right) \\
& N_{3}=C_{1}\left(B_{2}^{2}-2 B_{1} B_{3}\right) \\
& N_{4}=\left(\frac{B_{3}^{2}}{C_{4}}\right)\left(C_{1} C_{2}-C_{0} C_{3}\right) \\
& D_{1}=C_{1}\left(C_{2} C_{3}-C_{1} C_{4}\right)-C_{0} C_{3}^{2}
\end{aligned}
$$

when $y(\Omega)$ is in the general form as

$$
y(\Omega)=\frac{-B_{3} \Omega^{3}-B_{2} \Omega^{2}+B_{1} \Omega+B_{0}}{C_{4} \Omega^{4}-C_{3} \Omega^{3}-C_{2} \Omega^{2}+C_{1} \Omega+C_{0}}
$$

By equating equation (10) and the above equation to obtain $B_{i}$ and $C_{i}$ coefficients and using the analytical expression (equation (14)), the performance index can be defined as

$$
P I=\pi \frac{16 \eta^{4}+12 \eta^{2}+5}{4 \eta}
$$

Clearly, the optimal value of the damping at which the $\mathrm{H}_{2}$ norm is minimized can be calculated by differentiating equation (16) with respect to the damping $\eta$ and equating the derivative to zero

$$
\frac{d(P I)}{d \eta}=\pi \frac{48 \eta^{4}+12 \eta^{2}-5}{4 \eta^{2}}=0
$$

which yields

$$
\eta_{\text {opt }}=0.4702
$$

Interestingly, the optimal value of the target damping is constant for all primary systems. Figure 3 shows the $\mathrm{H}_{2}$ norm (equation (16)) as a function of the closed-loop

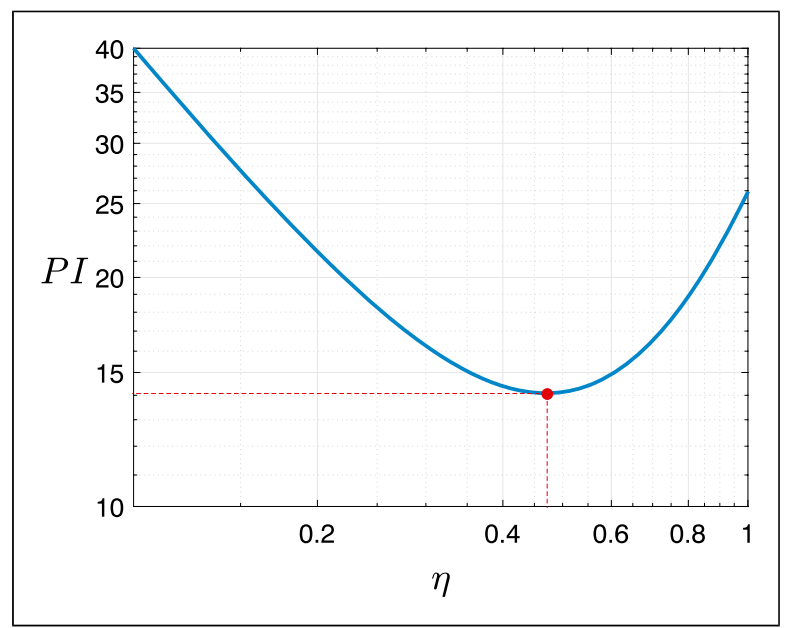

Figure 3. $\mathrm{H}_{2}$ norm of the controlled system for increasing the damping ratio of the closed-loop function $\eta$.

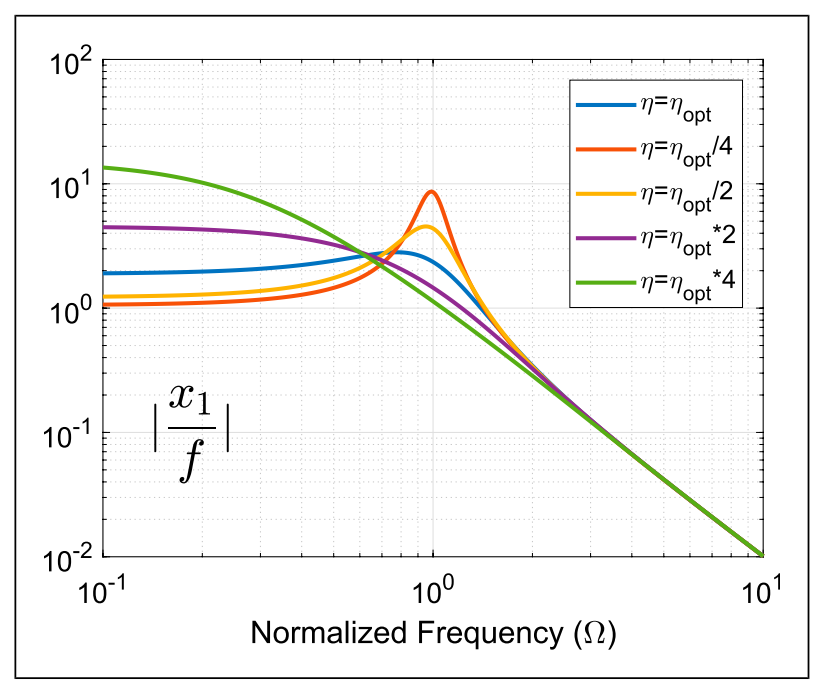

Figure 4. Frequency response function of the closed-loop system for different values of the target damping $\eta$.

damping ratio $\eta$. Amplitude reduction around the resonance frequency is responsible for the decreasing $\mathrm{H}_{2}$ norm from 0 to $47.02 \%$ of the target damping ratio. However, for higher damping ratio $\eta$, the increased magnitude at low frequency becomes more dominant than the amplitude reduction around the resonance. This can be seen in the frequency response shown in Figure 4 for five different values of the target damping defined as $\eta / \eta_{\text {opt }}: 1,(1 / 4)$, $(1 / 2), 2,4$.

\section{Influence of the PPF parameters}

In the following section, $P_{0}$ and $P_{1}$ are defined to be the pole of the primary system and the pole of the optimal PPF, respectively. The closed-loop pole when the optimal PPF is implemented is also shown by 


\section{I. The damping ratio of the PPF $(\xi)$}

Figure 5 shows the root-locus for various values of the damping ratio of the PPF $(\xi)$. Here, the other parameters of the controller, $\alpha$ and $\beta$, are set to the optimal values. In this section, $P_{2}$ and $P_{3}$ are specified to be the poles of the PPF when $\xi=0.5 \xi_{\text {opt }}$ and $\xi=2 \xi_{\text {opt }}$, respectively. In addition, $\bullet$ and $\boldsymbol{\Delta}$ indicate the closed-loop poles when $\xi=0.5 \xi_{\text {opt }}$ and $\xi=2 \xi_{\text {opt }}$, respectively. The locus consists of two loops, starting, respectively, from the pole of the primary system and the pole of the PPF. One of the loops goes to the real axis, and the other one goes to infinity. If $\xi<\xi_{\text {opt }}$, both closed-loop poles have less damping ratio than for the optimal case. In this case, one pole has higher and the other one has lower frequency than the resonance frequency of the primary system.

If $\xi>\xi_{\text {opt }}$, one pole is more damped than the other one. The heavily damped pole is placed close to the pole of the controller. Therefore, the pole/zero cancellation occurs because the zero of the closed-loop function (equation (6)) is the pole of the controller. As a consequence, the performance of the controller is degraded, and especially it is no longer effective if $\xi \rightarrow \infty$. It is worth pointing out that the value of the $H_{2}$ norm for $\xi=0.5 \xi_{\text {opt }}$ and $\xi=2 \xi_{\text {opt }}$ is about $29 \%$ and $21 \%$ greater than the optimum one, respectively.

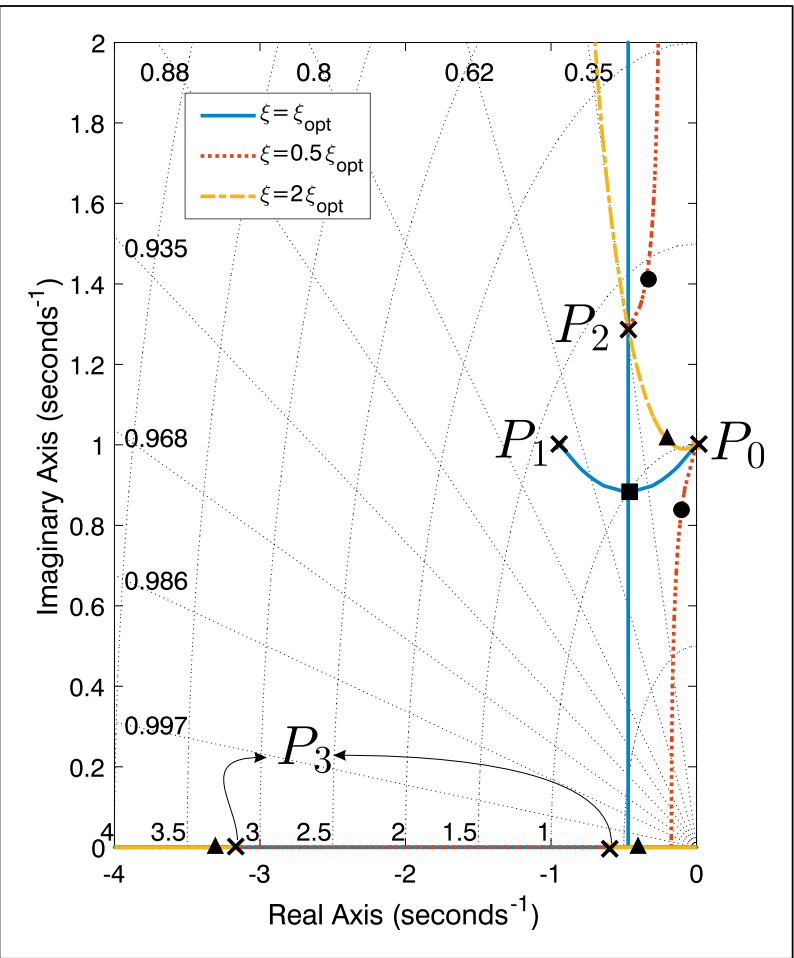

Figure 5. Root-locus for three different values of the damping ratio $\xi\left(P_{0}\right.$ : pole of the primary system; $P_{1}$ : pole of the PPF; $\mathbf{\square}$ closed-loop poles when $\xi=\xi_{\text {opt }} ; P_{2}$ : pole of the PPF; $\bullet$ : closed-loop poles when $\xi=0.5 \xi_{\text {opt }} ; P_{3}$ : pole of the PPF; $\mathbf{\Delta}$ : closed-loop poles when $\xi=2 \xi_{\text {opt }}$ ). PPF: positive position feedback.
It is meaningful to examine the performance of the PPF on the frequency response for different values of the damping ratio $\xi$. Figure 6 shows the frequency response for five different damping ratios defined as $\xi / \xi_{\text {opt }}: 1,0.1,0,10$ and $\infty$. One sees that all the curves are intersecting at two frequencies which are called fixed points. For the damping ratios lower than the optimal value, the primary resonance is transformed into one anti-resonance and two new resonances appear in the vicinity of the old one. An interesting point here is that the increased magnitude of the response at low frequencies disappears when the value of the damping increases $\left(\xi>\xi_{\text {opt }}\right)$. Explicitly, the coupled system behaves like the primary system with no additional damping when $\xi \rightarrow \infty$.

\subsection{The frequency of the PPF $(\alpha)$}

The root-locus plots for three different values of the frequency of the PPF are compared in Figure 7. In this case, the other control parameters $(\xi$ and $\alpha$ ) are set to the optimal values. In this case, $P_{2}$ and $P_{3}$ are ascertained to be the poles of the PPF when $\alpha=0.5 \alpha_{\text {opt }}$ and $\alpha=2 \alpha_{\text {opt }}$, respectively. Furthermore, $\boldsymbol{*}$ and $\boldsymbol{\Delta}$ demonstrate the closed-loop poles when $\alpha=0.5 \alpha_{o p t}$ and $\alpha=2 \alpha_{o p t}$, respectively. If $\alpha \neq \alpha_{o p t}$, one of the closed-loop poles is more damped than the other one. The heavily damped pole is less effective than the other pole because of the pole/zero cancellation as discussed in Section 3.1. It can be seen in equation (6) that if $\alpha \rightarrow 0$, the PPF could no longer add any damping to the system. Also, if $\alpha \rightarrow \infty$, the pole of the PPF becomes ineffective in terms of adding damping to the system. However, the other pole moves on the branch which goes toward the origin. This modifies the frequency of the pole to a slightly lower value. It should be noted that the $\mathrm{H}_{2}$ norm for $\alpha=0.5 \alpha_{\text {opt }}$ and $\alpha=2 \alpha_{\text {opt }}$ is $46 \%$ and $80 \%$ greater than the optimum one, respectively.

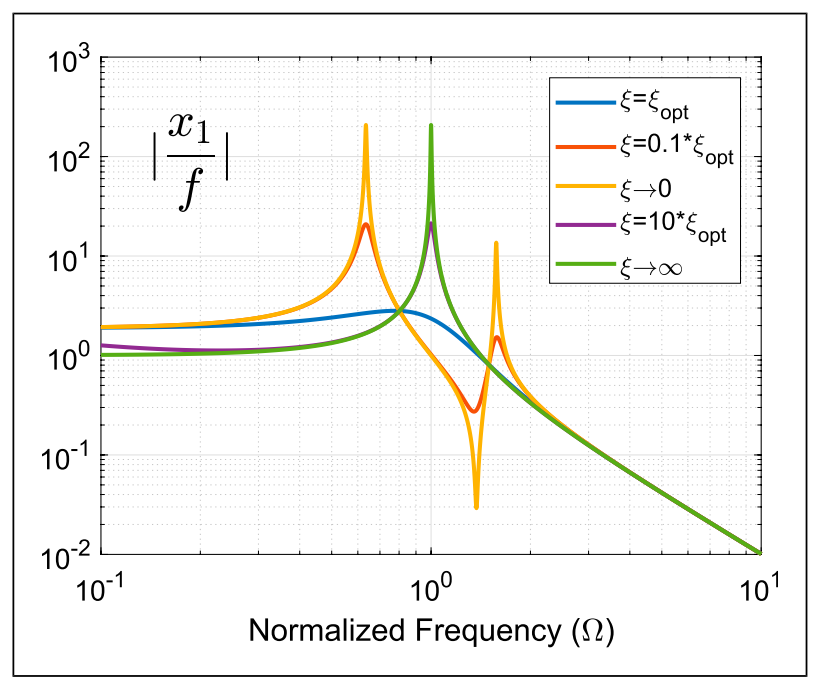

Figure 6. Frequency response of the controlled system for different values of the damping ratio $\xi$. 


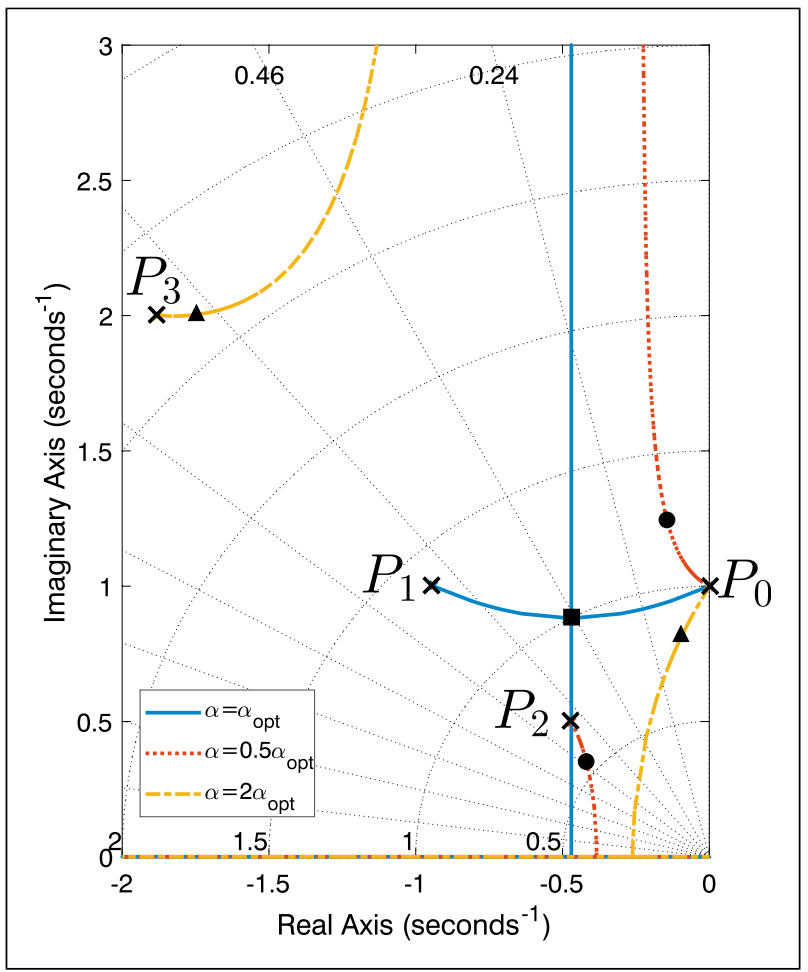

Figure 7. Root-locus for three different values of the frequency of the PPF $\alpha\left(P_{0}\right.$ : pole of the primary system; $P_{1}$ : pole of the PPF; closed-loop poles when $\alpha=\alpha_{o p t} ; P_{2}$ : pole of the PPF; $\bullet$ : closed-loop poles when $\alpha=0.5 \alpha_{o p t} ; P_{3}$ : pole of the PPF; $\boldsymbol{\Delta}$ : closed-loop poles when $\alpha=2 \alpha_{o p t}$ ). PPF: positive position feedback.

Figure 8 depicts the frequency response for five different values of $\alpha$. The evidence of the performance degradation can be seen when $\alpha \rightarrow 0$. In this case, the controlled response has the same dynamic behavior as the primary system with no damping. Two important observations are made when the frequency of the controller is larger than its optimal value. First, no more degradation of the static stiffness can be observed in the low frequency response of the system. Second, it results in a dynamically softer system. It is because the controller behaves like a positive constant gain in this case. This constant value amplifies the feedback signal which is positively proportional to the displacement. Consequently, it reduces the stiffness and the resonance frequency of the structure.

\subsection{The feedback gain $(\beta)$}

It is worth pointing out that the system remains stable even if the changes in the damping $\xi$ or the frequency $\alpha$ of the controller are significant. However, it is not the case for the feedback gain $\beta$. To check the stability, the Routh-Hurwitz stability criterion is used. For this purpose, consider $A_{0}, A_{1}$, $A_{2}, A_{3}$, and $A_{4}$ as the corresponding coefficients of the Laplace variable in the denominator of equation (6) from $s^{0}$ to $s^{4}$, respectively. This criterion states that the poles of

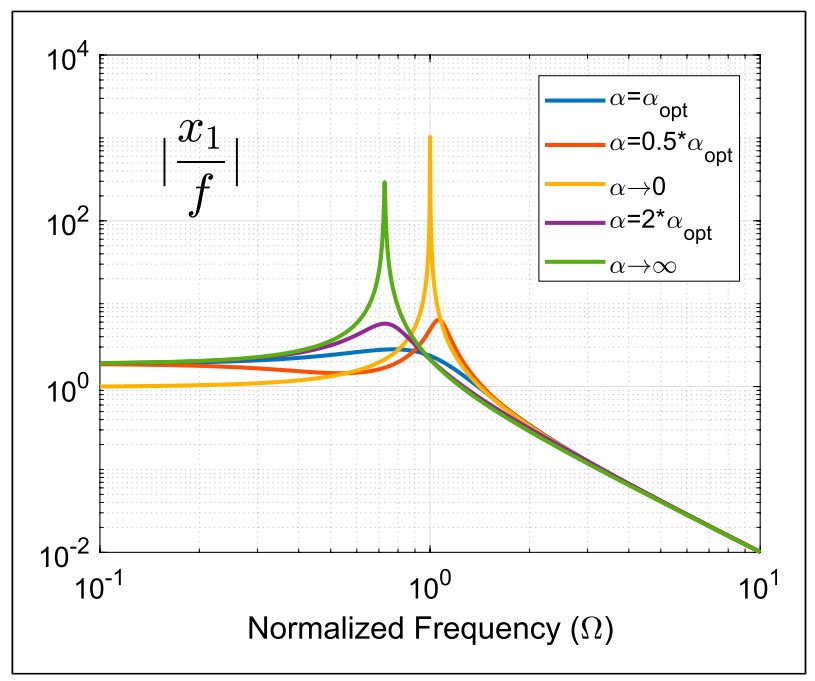

Figure 8. Frequency response of the controlled system for different values of the frequency of the positive position feedback $\alpha$.

equation (6) have negative real parts if and only if the following conditions are satisfied

$$
\begin{gathered}
A_{0}, A_{1}, A_{2}, A_{3}, A_{4}>0 \\
A_{2} A_{3}-A_{1} A_{4}>0 \\
A_{1} A_{2} A_{3}-A_{1}^{2} A_{4}-A_{0} A_{3}^{2}>0
\end{gathered}
$$

It can be derived that the system is stable if and only if the feedback gain $\beta$ is considered as

$$
0<\beta<1
$$

The designed optimal value of the feedback gain $\beta_{\text {opt }}$ is equal to 0.4693 , which ensures the stability of the system. The corresponding root-locus for three different values of the feedback gain is shown in Figure 9. The damping ratio $\xi$ and the frequency $\alpha$ are set to the optimal values. In this section, $P_{2}$ and $P_{3}$ are the poles of the PPF when $\beta=0.5 \beta_{\text {opt }}$ and $\beta=2 \beta_{\text {opt }}$, respectively. Moreover, $\bullet$ and $\boldsymbol{\Delta}$ show the closed-loop poles when $\beta=0.5 \beta_{\text {opt }}$ and $\beta=2 \beta_{\text {opt }}$, respectively. If $\beta<\beta_{\text {opt }}$, the heavily pole located closed to the pole of the controller becomes less effective because of the pole/zero cancellation explained in Section 3.1. Especially, equation (6) evidences that, if $\beta \rightarrow 0$, the controller is no longer effective to increase the damping of the system. If $\beta>\beta_{\text {opt }}$, the heavily damped pole can improve the damping of the closed-loop response. However, this pole moves directly toward the origin, which makes the system softer. The value of the $\mathrm{H}_{2}$ norm for $\beta=0.5 \beta$ and $\beta=2 \beta$ increases $45 \%$ and $292 \%$ with respect to the optimum case, respectively. Another interesting result is shown in the frequency response of the controlled system (Figure 10) for five different values of the feedback gain. 


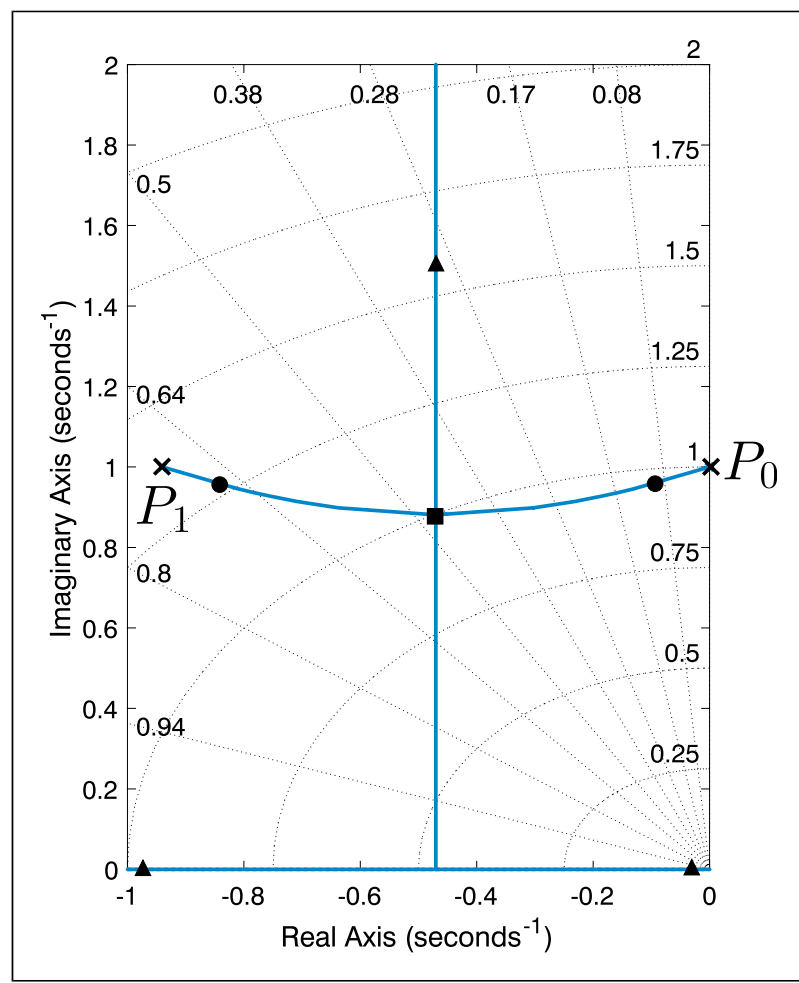

Figure 9. Root-locus for three different values of the feedback gain $\beta$ ( $P_{0}$ : pole of the primary system; $P_{1}$ : pole of the positive position feedback; $\mathbf{\square}$ : closed-loop poles when $\beta=\beta_{\text {opt }}$; $\bullet$ : closedloop poles when $\beta=0.5 \beta_{\text {opt }} ; \quad \boldsymbol{\Delta}$ : closed-loop poles when $\left.\beta=2 \beta_{\text {opt }}\right)$.

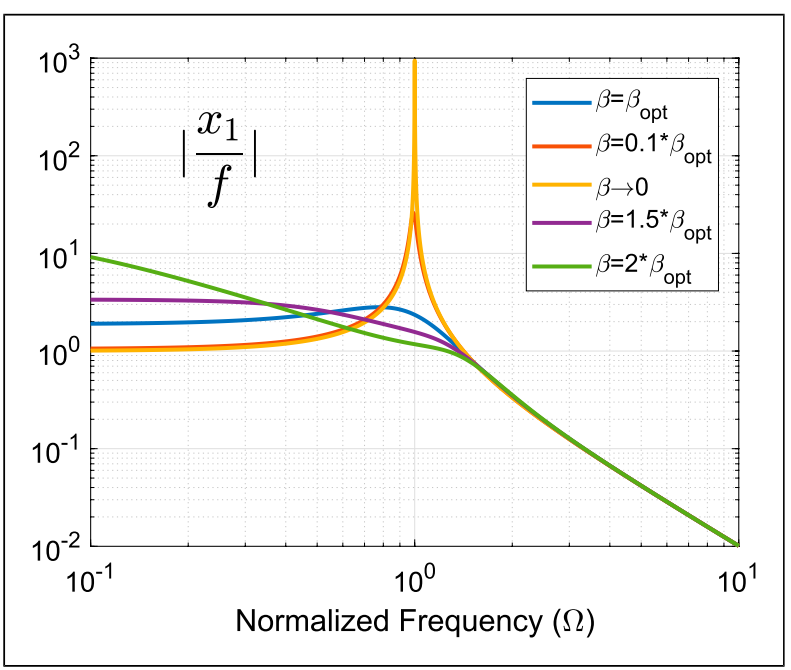

Figure 10. Frequency response of the controlled system for different values of the feedback gain $\beta$.

The control effectiveness degrades in terms of the resonance peak by decreasing the feedback gain. The more vibration attenuation of the resonance peak comes at the expense of the increased static stiffness when $\beta \approx 1$. This has been already discussed as a major problem in the previous section.

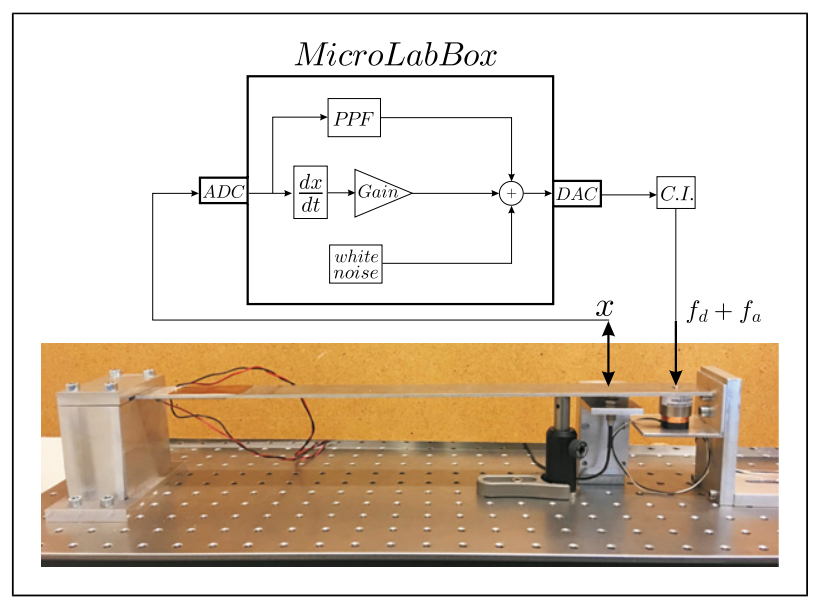

Figure II. Picture of the test benchmark used to test the proposed controller. Cantilever beam clamped at one end and equipped with a voice coil actuator and an eddy-current sensor at the other end (ADC: analog-to-digital-converter; DAC: digital-toanalog-converter; C.I.: current-injector).

\section{Experimental validation}

As shown in Figure 11, the experimental setup used to test the optimal proposed controller is a cantilever beam with the dimensions $455 \mathrm{~mm}, 40 \mathrm{~mm}$ and $3 \mathrm{~mm}$ as length, width and thickness, respectively. The resonance of interest is the first bending mode of the beam. According to the first mode shape, the maximum displacement occurs at the free-end of the beam. Thus, a voice coil actuator is placed there and an eddy-current sensor is fixed next to the actuator to measure the displacement motion. A dSPACE MicroLabBox is used for the purpose of both the data acquisition as well as the control system. The whole control scheme is designed first inside the graphical SIMULINK environment of MATLAB and then compiled. The compiled file is downloaded onto the ControlDesk environment of $d S P A C E$ software, which is connected directly to the MicroLabBox hardware, to be executed in real time. The control scheme is updated at a sampling frequency of $10 \mathrm{kHz}$, while the measured data are also recorded at the same sampling frequency. A current amplifier (ADD-45N) is used to drive the voice coil actuator.

Although the obtained formulas are strictly valid for an undamped primary system, they can fairly be used to specify the parameters of controllers for lightly damped structures as well. The beam shown in Figure 11 behaves like a lightly damped structure when the voice coil actuator is not mounted. The damping ratio in this case is $0.8 \%$. However, it becomes $8 \%$ when the actuator is mounted. This is because of the dissipation of the energy caused by the eddy current inside of the electromagnetic transducer. A negative damping force is implemented actively to compensate the undesired damping of the actuator. Displacement feedback with a positive derivative operator is used to 


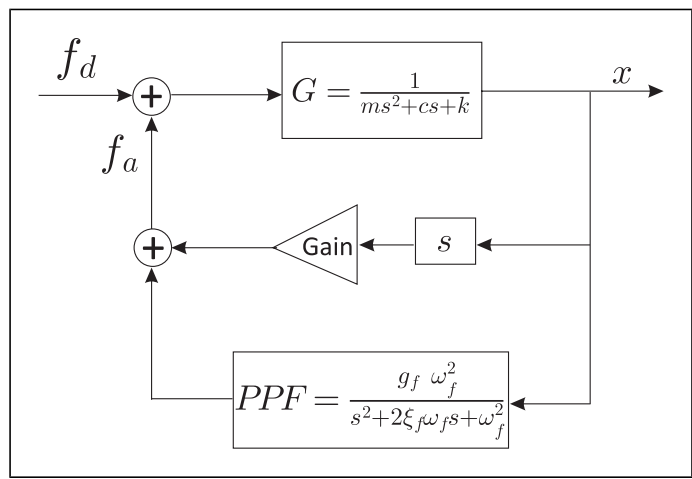

Figure 12. Block diagram of the controller architecture (G: primary system; c: damping of the primary system).

add the negative damping in the system. The configuration scheme for the experimental study is shown in Figure 11. A white noise generator is employed to excite the structure from $0 \mathrm{~Hz}$ to $100 \mathrm{~Hz}$ by using the same actuator in the setup. It also applies the control force. Furthermore, simulations are carried out to examine the performance of the designed optimal controller numerically. The block diagram of the control architecture is shown in Figure 12.

Figure 13 shows the frequency response functions from the disturbance force (the white noise) to the displacement of the eddy-current sensor. It also compares the simulations and the experiments without control and with the optimal PPF. The values of the stiffness and the resonance obtained by matching one DOF system to the experimental result are $295 \mathrm{~N} / \mathrm{m}$ and $8.65 \mathrm{~Hz}$, respectively. The effective structural damping after addition of negative damping is found to be $0.45 \%$. An interesting observation is that the experimental result shows a good agreement with the simulations. The maximum amplitude of the coupled response is reduced almost 33 times compared to the primary system. Also, amplification of the response at low frequencies is only about $88 \%$, which is not detrimental. As an important consequence, the result confirms that the obtained formula is also valid for the lightly damped structure.

To better understand the stability of the closed-loop system in terms of the phase and the gain margins, the loop-gain of the system is also shown in Figure 14. It is obtained by multiplying the primary system to the designed optimal controller (equation (21a)). According to Figure 14, the phase is always bounded between $180^{\circ}$ and $-180^{\circ}$ in the entire frequency range, which ensures the stability of the closed-loop system. The gain margin is $6.57 \mathrm{~dB}$ obtained when the phase touches the $180^{\circ}$ at $0 \mathrm{~Hz}$. Furthermore, the minimum phase margin is $-46.7^{\circ}$ where the magnitude of response touches the $0 \mathrm{~dB}$ line.

$$
\text { Loop-gain: }-G \times P P F
$$

$$
\text { Sensitivity: } \frac{1}{1-G \times P P F}
$$

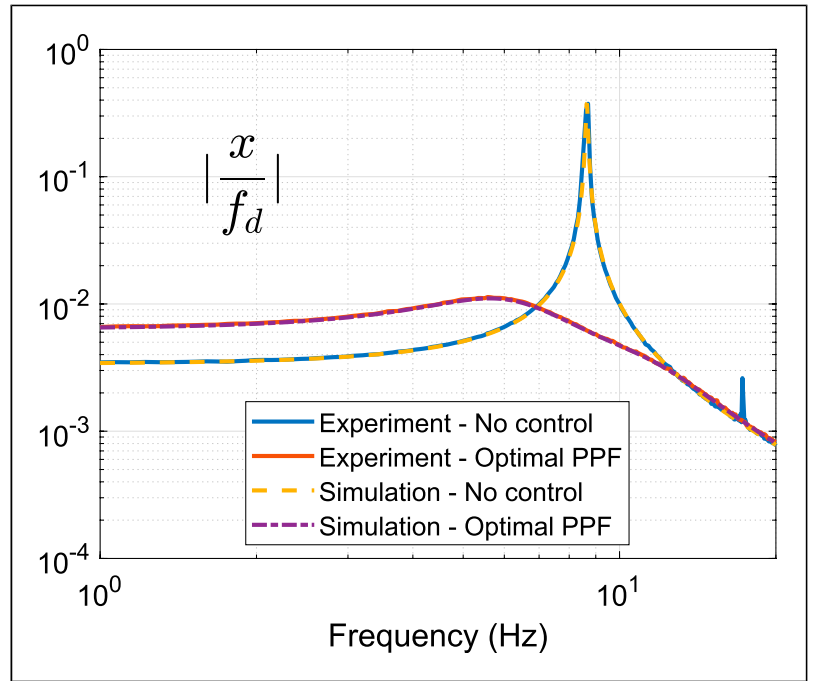

Figure 13. Frequency response functions from the disturbance force to the displacement of the eddy-current sensor obtained from the simulations and experiments with and without optimal positive position feedback controller.

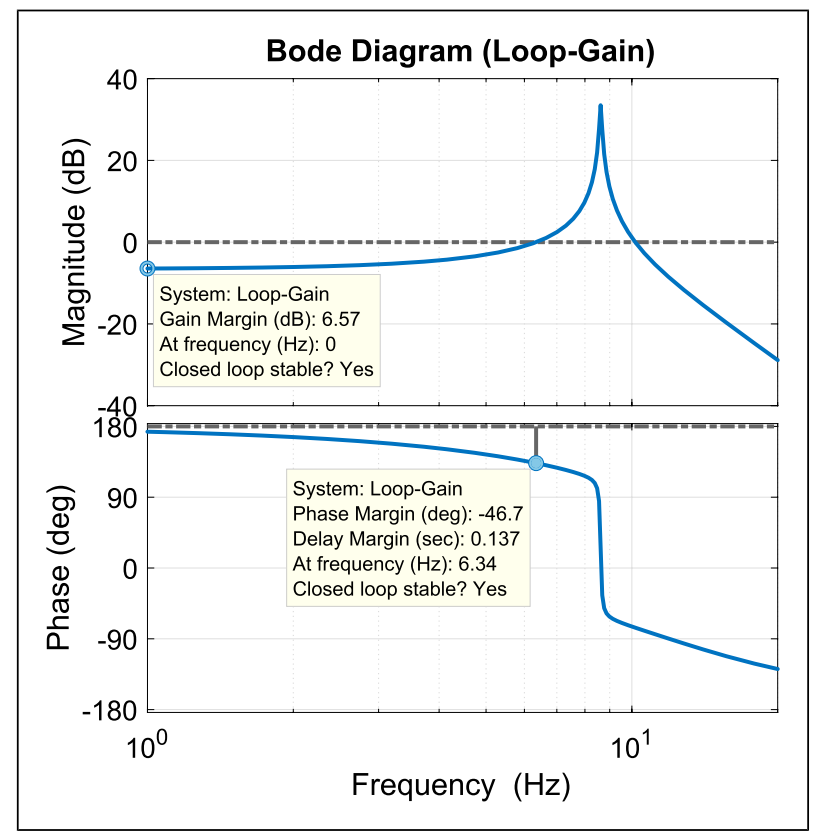

Figure 14. Bode diagram of the loop-gain for the designed optimal positive position feedback controller $(\bigcirc$ : gain margin; phase margin).

The sensitivity function (equation (21b)) is also depicted in Figure 15. As it is expected, the controller amplifies the disturbance of the system below the crossover frequency i.e. when the magnitude of response intersects the $0 \mathrm{~dB}$ line. This leads to increasing the static stiffness of the system. After the crossover frequency, the controller reduces the disturbance especially around the resonance frequency 


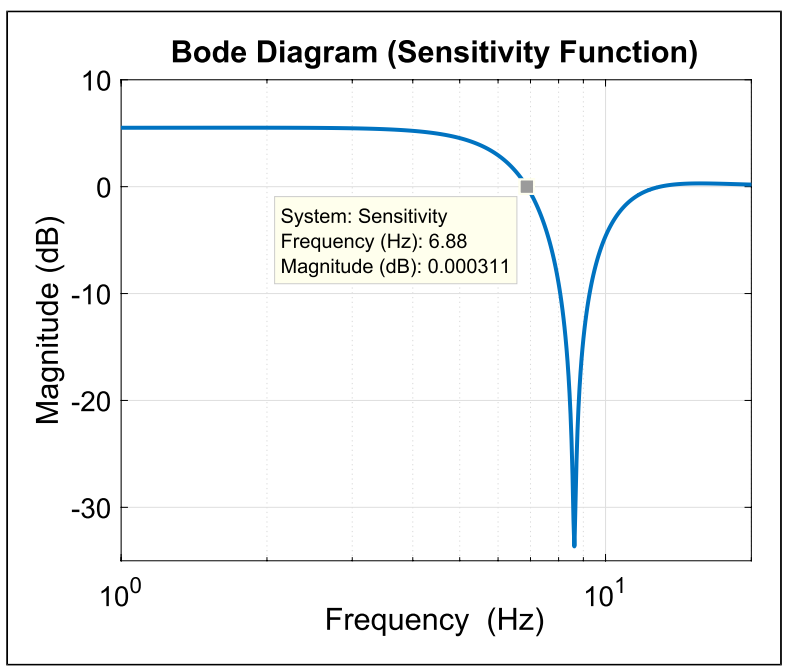

Figure 15. Sensitivity curve for the designed optimal positive position feedback controller (匹: crossover).

where the maximum reduction occurs. At high frequency, the controller is no longer effective.

\section{Conclusion}

An easy to implement optimal PPF control procedure has been proposed, analyzed and experimentally implemented in this paper. The trade-off between the amplitude reduction at the resonance frequency and the amplification of response at the frequencies lower than the tuning frequency has been highlighted and used to optimize the PPF controller. To this end, the position of the poles of the closed-loop system is determined using the maximum damping criterion. Then, the parameters of the PPF controller could be expressed as a function of the target damping in the closed-loop system. The optimal value of the target damping was evaluated using $\mathrm{H}_{2}$ optimization. It has also been demonstrated, through sensitivity analysis, that the performance of the system degrades if the parameters of the PPF are deviated from the optimal values. From the experimental tests, we could observe that the obtained formula can be effective to calculate the optimal PPF for the lightly damped primary system as well. The extension of the proposed optimal PPF to damping several modes of the primary structure is the topic of future work.

\section{Declaration of conflicting interests}

The author(s) declared no potential conflicts of interest with respect to the research, authorship, and/or publication of this article.

\section{Funding}

The author(s) disclosed receipt of the following financial support for the research, authorship, and/or publication of this article:The authors are grateful to the financial support of MAVERIC (Wal'innov project 1610122).

\section{ORCID iDs}

Ahmad Paknejad (D) https://orcid.org/0000-0003-2920-144X

Michel Osée (D) https://orcid.org/0000-0003-0487-7590

\section{References}

Balas MJ (1979) Direct velocity feedback control of large space structures. Journal of Guidance and Control 2(3): 252-253.

Crandall SH and Mark WD (1963) Random Vibration in Mechanical Systems. MA, USA: Academic Press.

Dosch JJ, Inman DJ and Garcia E (1992a) A self-sensing piezoelectric actuator for collocated control. Journal of Intelligent Material Systems and Structures 3(1): 166-185.

Dosch JJ, Leo DJ and Inman DJ (1992b) Comparison of vibration control schemes for a smart antenna. In: Proceedings of the $31 \mathrm{st}$ IEEE conference on decision and control, Tucson, AZ, 16-18 December 1992. Tucson, AZ: IEEE.

Eaton DCG (1997) An overview of structural acoustics and related high-frequency-vibration activities. ESA Bulletin-European Space Agency 92: 89-94.

Fagan GT (1993) An experimental investigation into active damage control systems using positive position feedback for $A V C$. Master's Dissertation, Virginia Tech, Virginia.

Fanson JL (1987) An experimental investigation of vibration suppression in large space structures using positive position feedback. PhD Thesis, California Institute of Technology, California.

Fenik S and Starek L (2008) Optimal PPF controller for multimodal vibration suppression. Journal of Engineering Mechanics 15(3): 153-173.

Fleming AJ, Behrens S and Moheimani SOR (2000) A new approach to piezoelectric shunt damping. Proceedings of International Symposium on Smart Structures and Microsystems.

Fuller CR and von Flotow AH (1995) Active control of sound and vibration. IEEE Control Systems Magazine 15(6): 9-19.

Goh CJ (1983) Analysis and control of quasi distributed parameter systems. PhD Thesis, California Institute of Technology, California.

Inman DJ (2001) Engineering Vibration. Englewood Cliffs, NJ: Prentice-Hall.

Kwak MK and Han S-B (1998) Application of genetic algorithms to the determination of multiple positive-position feedback controller gains for smart structures. In: Proceedings SPIE 3323, smart structures and materials 1998: Mathematics and control in smart structures, San Diego, CA, 24 July 1998. San Diego, CA: SPIE.

Lazan BJ (1954) Fatigue failure under resonant vibration conditions. Report no. 54-20. MN, US: University of Minnesota.

Marinangeli L, Alijani F and HosseinNia SH (2018) Fractionalorder positive position feedback compensator for active vibration control of a smart composite plate. Journal of Sound and Vibration 412: 1-16.

McEver MA (1999) Optimal vibration suppression using on-line pole/zero identification. Master's Thesis, Virginia Tech, Virginia.

Naji S, Çelik OC, Johnson Alengaram U, et al. (2014) Structure, energy and cost efficiency evaluation of three different lightweight 
construction systems used in low-rise residential buildings. Energy and Buildings 84: 727-739.

Niu W, Li B, Xin T, et al. (2018) Vibration active control of structure with parameter perturbation using fractional order positive position feedback controller Journal of Sound and Vibration 430: 101-114.

Osinski Z (2018) Damping of Vibrations. Boca Raton, FL: CRC Press.

Oude Nijhuis MHH (2003) Analysis tools for the design of active structural acoustic control systems. PhD Thesis, University of Twente, Netherlands.
Pota HR, Reza Moheimani SO and Smith M (1999) Resonant controllers for flexible structures. In: Proceedings of the 38th IEEE conference on decision and control, Phoenix, AZ, 7-10 December 1999. Phoenix, AZ: IEEE.

Preumont A, Dufour J-P and Malekian C (1992) Active damping by a local force feedback with piezoelectric actuators. Journal of Guidance, Control, and Dynamics 15(2): 390-395.

Wilby JF and Pope LD (1980) Prediction of the acoustic environment in the space shuttle payload bay. Journal of Spacecraft and Rockets 17(3): 232-239. 